\title{
Protection of CT suites from COVID-19 infection in a tertiary emergency hospital
}

\author{
Tetsuya Katsumori $^{1} \cdot$ Tatsuya Yoshikawa $^{1}$
}

Received: 20 March 2020 / Accepted: 23 March 2020 / Published online: 4 April 2020

(c) Japan Radiological Society 2020

\section{To the Editor}

We read with great interest an article by Nakajima et al. entitled "COVID-19 pneumonia: infection control protocol inside computed tomography suites" published in the Japanese Journal of Radiology [1]. Rapidly increasing are publications regarding the diagnosis of COVID-19 pneumonia by computed tomography (CT), but limited articles seemingly have reported infection control in the CT suites. We would like to thank Nakajima et al. for publishing a good and timely article focusing on this critical issue. We want to comment on the protection of CT suites from COVID-19 infection in our tertiary emergency hospital in Japan. Approximately 10-15 patients suspected of COVID-19 infection are being transferred to our hospital each day at the time of writing this manuscript (March 19th, 2020). Our hospital has two CT suites; one is used for scanning scheduled patients or performing interventional radiology under CT guidance, while the other is used for scanning emergency patients.

At our hospital, we do not perform CT to screen for or as a first-line test to diagnose COVID-19 in patients with COVID-19 infection confirmed or suspected. This is because CT findings of COVID-19 are not seemingly specific and overlap with other infections [2]. Despite several limitations, viral tests, including reverse-transcription polymerase chain reaction (RT-PCR), are currently used as a diagnostic indicator worldwide [3]. Instead of CT, we consistently take a chest radiograph with portable radiography in the specific rooms. Our strategies are consistent with the current American College of Radiology (ACR) recommendations [3]. Additionally, our approach reduces the risk of COVID-19 infection in the CT suites.

Tetsuya Katsumori

katsumo@eurus.dti.ne.jp

1 Department of Radiology, Saiseikai Shiga Hospital, Ohashi 2-4-1, Ritto, Shiga 520-3046, Japan
On the other hand, if patients with COVID-19 infection confirmed or suspected suffer from acute or serious diseases (e.g., stroke, fatal bleeding, and acute abdomen), CT will be performed. Under these conditions, the protection of CT suites from COVID-19 infection is critical for preventing the hospital-related transmission of COVID-19. We consider the infection control protocol reported by Nakajima et al. to be helpful [1].

Of importance is cleaning the CT suites after scanning patients with COVID-19 infection confirmed or suspected. This is because we need to use the CT suites for the next patients as soon as possible. Nakajima et al. reported that CT examinations on the patients with COVID-19 confirmed or suspected were performed at the end of the daily CT schedule [1]. Therefore, we estimate that their interval time may be approximately less than $12-18 \mathrm{~h}$ from scanning the patients with COVID-19 infection confirmed or suspected to scanning the next patient. The National Institutes of Health (NIH) reported that novel coronavirus was detectable in aerosols for up to $3 \mathrm{~h}$, up to $24 \mathrm{~h}$ on cardboard and up to 2-3 days on plastic and stainless steel [4]. Since unfortunately, our CT suites do not include a negative pressure ventilation system, we may need to take much longer intervals in our clinical practice. If we are to use CT for rapidly increasing patients with COVID-19 infection confirmed or suspected, we presume that CT operating times, as well as numbers of patients that can be scanned, will markedly reduce due to the extensive time spent on cleaning $\mathrm{CT}$ suites. In the worst-case scenario, healthcare using CT may collapse in our hospital.

The outcomes of any trials are worth verifying. Nakajima et al. [1] did not provide the number of patients (with COVID-19 infection confirmed or suspected) who underwent CT with the protection protocol. Did they successfully prevent hospital-related transmission of COVID-19 in all cases?

Finally, radiologists must protect against the transmission of COVID-19 infection not only in the CT suites, but 
also in other radiology areas, including MR suites, angiography suites, RI suites and radiation therapy suites. This is because each suite will be potentially used for patients with COVID-19 infection confirmed or suspected. Further discussions and information on the protection from COVID-19 infection are urgently needed.

Funding None.

\section{Compliance with ethical standards}

Conflict of interest The authors declare that they have no conflict of interest.

Ethical statement This article does not contain any studies with human participants or animals performed by any of the authors.

\section{References}

1. Nakajima K, Kato H, Yamashiro T, et al. COVID-19 pneumonia: infection control protocol inside computed tomography suites. Jpn J Radiol. 2020. https://doi.org/10.1007/s11604-020-00948-y.
2. Kanne JP, Little BP, Chung JH, Elicker BM, Ketai LH. Essentials for radiologists on COVID-19: an update-Radiology Scientific Expert Panel. Radiology. 2020. https://doi.org/10.1148/radio 1.2020200527.

3. ACR Recommendations for the use of Chest Radiography and Computed Tomography (CT) for Suspected COVID-19 Infection; 2020. https://www.acr.org/Advocacy-and-Economics/ACR-Posit ion-Statements/Recommendations-for-Chest-Radiography-andCT-for-Suspected-COVID19-Infection

4. New coronavirus stable for hours on surfaces SARS-CoV-2 stability similar to original SARS virus; 2020. https://www.nih.gov/ news-events/news-releases/new-coronavirus-stable-hours-surfaces

Publisher's Note Springer Nature remains neutral with regard to jurisdictional claims in published maps and institutional affiliations. 